\title{
Korean Wave en América Latina como resultado del multiculturalismo
}

\section{The Korean Wave in Latin America as a result of multiculturalism}

\section{>> Valentina Suyai}

Pontificia Universidad Católica de Chile vefigueroa@uc.cl

Orcid: https://orcid.org/0000-0002-5831-8455

Recibido: 7 de septiembre 2020.

Aprobado: 9 de octubre 2020.

Publicado: 16 de diciembre 2020

\section{Resumen}

En América Latina la revolución de la cultura popular surcoreana surge como un paradigma sociocultural, que se soporta en el éxito de la economía de esta potencia asiática. El escenario global, ya desde la era fordista del capitalismo, ha brindado herramientas claves para la distribución y maximización del mercado, dando como resultado la aparición del multiculturalismo como una nueva versión de la globalización del capital. Bajo este panorama, el presente trabajo estudia cómo el Hallyu se ha consolidado en Latinoamérica bajo la protección del capitalismo y la diversificación de las culturas.

\section{Palabras Clave:}

Capitalismo, multiculturalismo, Korean Wave, Latinoamérica, imagen, identidad. 


\section{Abstract}

The popular South Korean-culture revolution in Latin America was brought about as a social and cultural paradigm supported by the economic success attained by the Asian power. Since the introduction of capitalist Fordism, international context has provided key tools for market distribution and maximization, leading to the emergence of multiculturalism as a new version of capital globalization. This paper examines Hallyu's consolidation in Latin America, fueled by capitalism and cultural diversity.

\section{Keywords:}

Capitalism, Multiculturalism, Korean Wave, Latin America, Image, Identity.

\section{Introducción}

En el presente ensayo, se busca estudiar las razones por las cuales la imagen del Korean Wave, o Hallyu (en coreano), se ha convertido en un referente tan sólido en América Latina. El estudio contempla teorías como la del multiculturalismo (Zizek, 1997; Jameson, 1993); el modelo de cortesía de Brown y Levinson (1978); estudios sobre las fronteras (Jessop, 2004; Dilla y Breton, 2015); literatura sociológica y filosófica para explicar las mutaciones de los conceptos identidad/apariencia en la esfera pública (Mijaíl Bajtín, 1997; Hanna Arendt, 1958; Gianni Vattimo, 1989); y diversos textos académicos sobre el alcance del Korean Wave en la región. Estos estudios son necesarios para entender el orden en que se plantea la taxonomía de este trabajo. El propósito es explicar, mediante una metodología analítica, el actuar del ser humano en la economía moderna, para dar cuenta de la relación innegable entre 'mercado, sociedad e individuo'. Estos conceptos, como claves en la esfera pública, han sido históricamente manipulados por el capital y la economía mundo, ocasionando cambios en la vida de las personas y modificando sus 'necesidades' a través del tiempo. En este contexto, el desarrollo económico de Corea del Sur es un factor crucial para comprender el poder que existe tras la imagen del Hallyu.

Es preciso puntualizar que cuando se habla de imagen, se hace referencia a lo entendido como face en el modelo de cortesía propuesto por Brown y Levinson (1987); es decir, se refiere a la imagen pública creada por los hablantes al momento de actuar en una situación comunicativa. Esta construcción del sujeto social permite profundizar en el análisis del actuar humano en su interacción con otro, pues la protección de la imagen es un esfuerzo primordial en la comunicación. La salvaguarda de esta face, como lo entiende la sociolingüística, implica una consciencia social previa al acto de habla, ya que los hablantes se sitúan en un contexto determinado, con reglas tácitas propias de todo acto comunicativo. En relación a este fenómeno pragmalinguístico, y también social, se debe entender que el individuo moderno se desenvuelve en un espacio social y geopolítico, amparado por conductas que respetan una serie de normas sociales. Por eso, en la interacción entre el yo y el otro, se ponen en evidencia dos elementos constituyentes de la identidad del sujeto moderno; por un lado, la imagen social que los participantes desean mostrar, por otro lado, las normas implícitas que los hablantes respetan en ese esfuerzo por salvaguardar su face.

La llegada paulatina del mercado surcoreano a Latinoamérica fue un proceso planeado desde la década de los años noventa (Shim, 2008). La exportación de material cultural como programas televisivos, animación, telenovelas y películas fue parte de una primera llegada de este fenómeno. Sin embargo, el desplome repentino ocurrido a partir del 2010 es un 


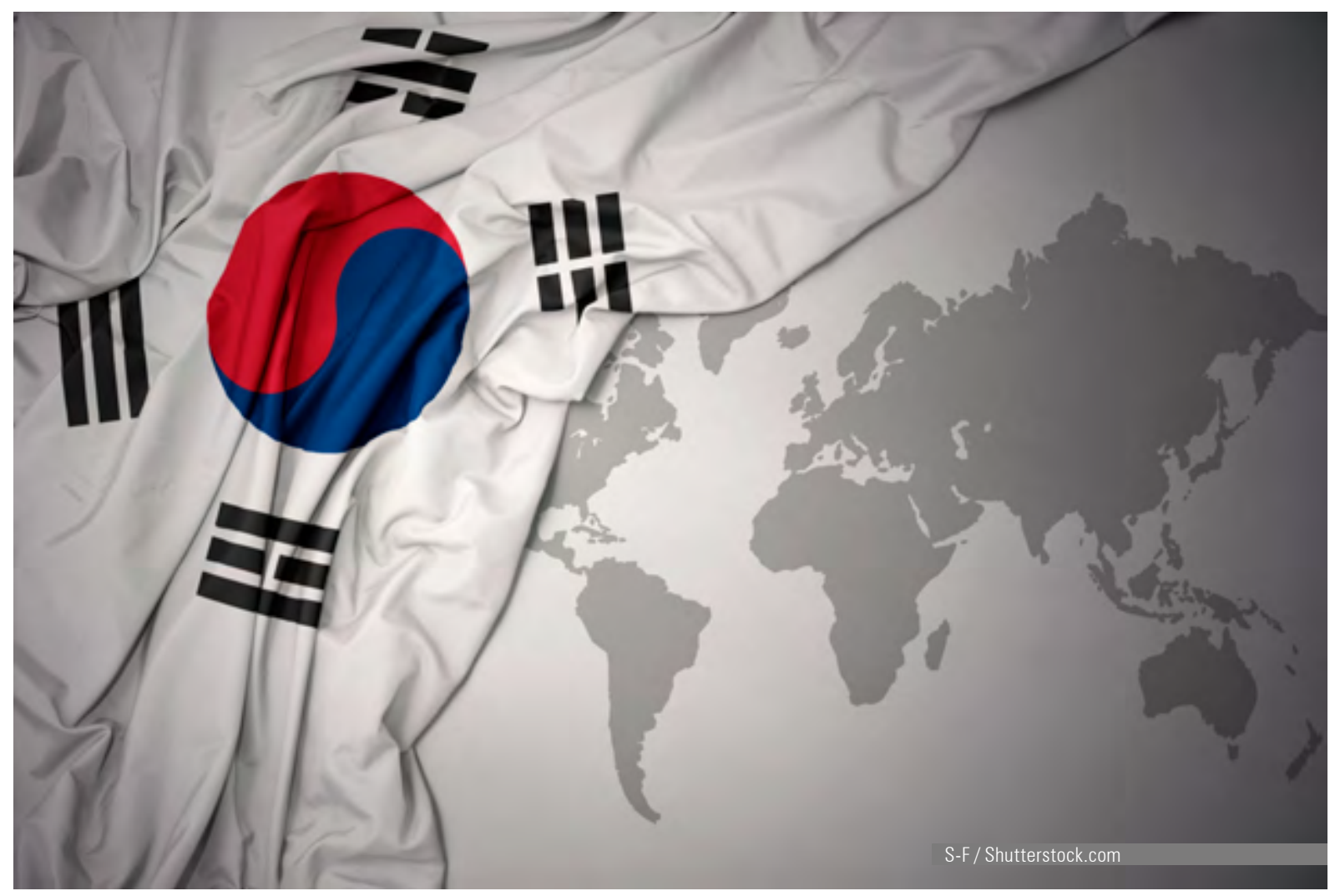

acontecimiento que aún cuesta trabajo comprender. El poder de los medios masivos de comunicación, junto al desarrollo de una época de transición de los grandes ejes económicos mundiales, propiciaron un escenario de solvencia indistinguida de la información. La población, dotada de un espacio en el que cualquier hablante se vuelve semejante a otro, comienza a gozar de un nuevo poder comunicativo, y a la vez de creación de la apariencia. En este sentido, el presente estudio pretende indagar en la literatura que devela los cambios modernos que ha producido el capitalismo desde su época fordista hasta la reciente expansión surcoreana en el mercado.

Con el fin de esclarecer la relación del trinomio propuesto (mercado, sociedad e individuo), el estudio comienza por indagar en antecedentes históricos marcados por el capitalismo. La importancia de la imagen nacional y el rol de su proyección en el mercado internacional.

Bajo la idea de que el mundo ha adoptado una disposición en pos del irreversible triunfo del nuevo modelo económico, las diversas disputas por el liderazgo global deben ser analizadas no solo como antecedente histórico, sino también como punto de partida de la ramificación constante de la cultura. Las disputas por el poder son protagonizadas por las superpotencias, dejando a las pequeñas economías reducidas a decisiones de lealtad y apoyo diplomático. En un contexto tan frívolo e inapelable, solo el arte es capaz de revelarse dentro del esquema, pues este siempre ha existido dentro del sistema como el único factor cuyos límites son tan difusos como sus predicciones al actuar. 


\section{Preguntas}

Las preguntas de este estudio se dividen en dos categorías: preguntas generales y preguntas específicas.

Las preguntas generales corresponden a: ¿Cómo influye el capitalismo en la construcción de imagen nacional para Corea del Sur? ¿De qué manera el Korean Wave se hace presente en América Latina?

Las preguntas específicas son: ¿Cómo se relaciona el multiculturalismo con la expansión del mercado surcoreano en América Latina? ¿La imagen de los seguidores del Hallyu responde a una nueva construcción de identidad?

\section{Discusión bibliográfica}

\subsection{El rol de los Estados en el proceso de mundialización del capital}

Se podría pensar que el liberalismo nació siendo 'antinacionalista', estableciéndose una dualidad entre las fuerzas privatizadoras del comercio y la figura del Estado. Sin embargo, antes de la Primera Guerra Mundial, fue el nacionalismo el que provocó el derrumbe del socialismo en la Unión Soviética. Vale decir, lo que triunfó fue la propuesta de lucha en contra de otros Estados, antes que la lucha en contra la burocracia nacional. Fueron las fronteras y los límites en la supremacía del poder los que motivaron el actuar desenfrenado de la humanidad durante el siglo XX. Y el capital, como combustible de la máquina humana, fue evolucionando a medida que las ideas de la acumulación mutaban en el entonces llamado 'primer mundo'. El fordismo fue el proyecto en el que mejor lució el capitalismo; el consumo de masas y la dirección de las sociedades hacia el mercado interno nacional fueron las bases para el régimen de estandarización y acumulación. En esta etapa es en la que se pueden plantear los procesos de culminación y consagración de los Estados Nacionales. A su vez, en América Latina muchos Estados adoptaron el modelo sustitutivo de importaciones durante los periodos populistas. La impresión de este modelo llamó la atención en todo el mundo, en especial tras la derrota del proyecto comunista en Rusia. Situación que acarreó procesos tácitos de desterritorialización y reterritorialización debido a las etapas de inclusión y exclusión del modelo. Finalmente, nace la vertiente neoliberal del capitalismo, el postfordismo. En esta instancia, se empieza a hablar del fenómeno de globalización del mercado, y de su elitización.

La República de Corea, hacia el fin de la guerra civil (1953), comienza a establecer cambios en la coalición doméstica. Stanley (2009) menciona tres categorías que propiciaron el fin del conflicto: en primer lugar, un sistema de políticas basadas en una moral ideológica. En segundo lugar, la política doméstica. Y en tercer lugar, las negociaciones establecidas como estrategias durante la guerra. Bajo estas premisas, el poder del Estado resulta necesario para implementar regulaciones e imposiciones durante las primeras décadas de la república. Los costos del conflicto civil produjeron consecuencias económicas y geopolíticas muy graves, asunto que condujo al Gobierno central a poner énfasis en el desarrollo económico. Kim Won Ho (2015) destaca la importancia que tuvo la educación en el modelo económico coreano. El autor enfatiza en el liderazgo del Estado y en la actuación de los chaebol en el mercado: "su política (de Corea del Sur) educativa se ajustó para facilitar el desempeño de las industrias nacionales" (p. 207). Las personas, entendidas como recursos laborales y capitales humanos, tuvieron que ajustarse a las medidas autoritarias con el fin de obtener el logro económico del país. El emblema del desarrollo se convirtió en el propósito del Estado. En cuanto a los ciudadanos, quienes aún poseían un fuerte valor del nacionalismo por las 


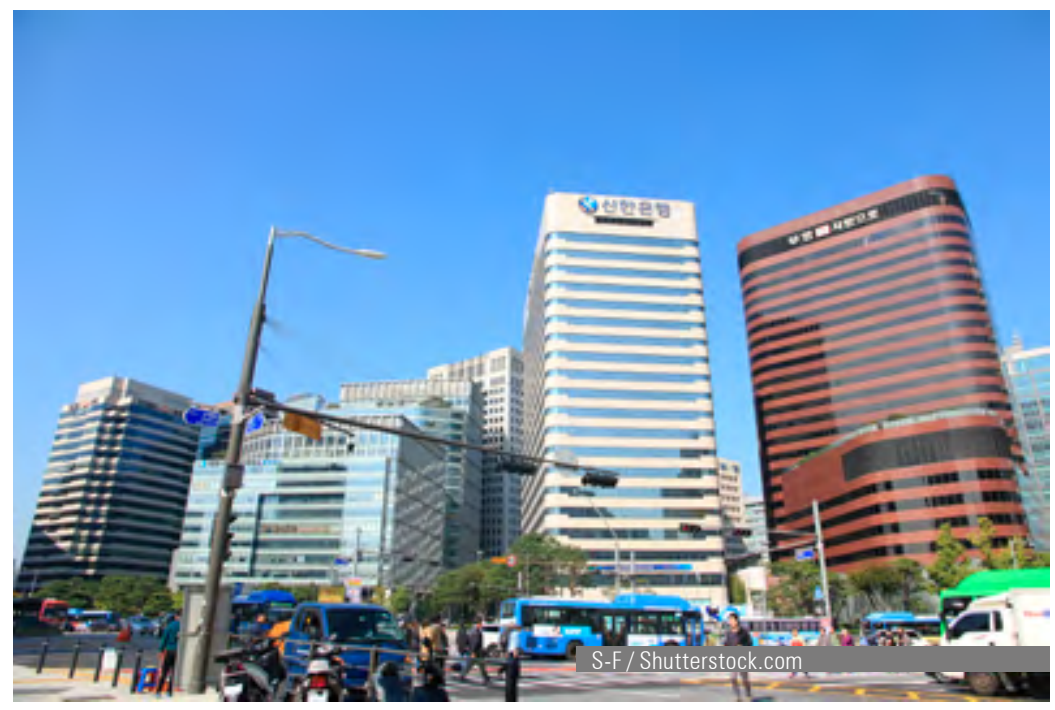

circunstancias geopolíticas, eran reprimidos por el régimen militar, y manejaban escasos recursos para detener ese actuar.

Corea del Sur logra integrarse a las economías motoras de la era de la globalización. Los chaebol, dotados de sus privilegios en la sociedad y economía coreana, se convirtieron en figuras importantes a escala internacional; consiguiendo un incremento de 22\% en el PIB per cápita entre 1963 y 1999, lo que se traduce en un resultado de 27\% hacia fines del año 1999 (Pipitone, 2003, p. 328). Luis Riffo (2003), respecto a la globalización, destaca el proceso de transformación de la organización del trabajo; el autor asegura que las nuevas estrategias de flexibilidad en contratación, despido y uso de los trabajadores, fueron provocando el retiro del Estado en varias funciones de apoyo social y comunitario (p. 25). En el caso de América Latina, Riffo (2003) señala que en la era de la globalización ocurre una doble dinámica en las áreas metropolitanas. Por un lado aparecen "nuevas dinámicas económicas asociadas a sectores económicos emergentes, mientras que por otra parte ha provocado un incremento de las desigualdades" (p. 25). La apertura del comercio, si bien trajo severas consecuencias sociales a los Estados de América Latina, produjo también un fuerte crecimiento de la inversión directa extranjera. Las ciudades comenzaron a internacionalizarse, y los citadinos empezaron a consumir productos y cultura de diferentes partes del mundo. En este nuevo panorama, las fronteras son entendidas a través de diversas ideas que parecieran chocar entre sí.

\subsection{Fronteras e intercambio desigual en el orden capitalista}

Dilla y Breton (2018), discuten el contraste entre dos acepciones diacrónicas del término frontera. En primer lugar, se encuentran las fronteras westfalianas, que son entendidas como los "límites rígidos que maduraron en los Estados nacionales" (p. 16). Su uso hermético y protector ayudó a garantizar la hegemonía de los gobiernos, separándolos de fuerzas externas que amenazaban a las naciones. Y, en segundo lugar, los autores mencionan que las fronteras son "líneas que cruzan los factores de intercambio económico" (p. 16). En este caso, se hace referencia a las experiencias europeas durante la mundialización del mercado, y al acuerdo de libre comercio norteamericano. La dicotomía expuesta por Dilla y Breton (2018) surge en un contexto de problematización en torno al concepto de fronterización de los Estados. La necesidad de la frontera ha ido adaptándose a los escenarios; sin embargo, 
prevalece la idea de diferenciación, es decir, las fronteras "se articulan como mecanismos de disciplinarización/normalización de la diferencia" (p. 18). Dicha característica — según los autores- propicia el escenario para el intercambio desigual.

El intercambio desigual es un requerimiento imperativo en la movilización de las fronteras. La necesidad/demanda de una parte es clave para la otra; y dicha necesidad, situada en una precariedad prolongada, es la condición en la que se encuentra América Latina. Norma de los Ríos (1998), en su estudio sobre Bagú (1997), pone en discusión el escenario desequilibrado en el cual se encuentran las naciones latinoamericanas. Esta condición, como parte de la norma del modelo capitalista, obliga a "mantener un porcentaje alto de la población dentro de límites rigurosos de ingresos" ( $p$. 21). Casos como Arica-Tacna en Chile y Perú; Tijuana-San Diego en México y Estados Unidos; Cúcuta-San Cristóbal en Venezuela y Colombia, son algunos ejemplos de la gran escala de movilidad humana y comercial que se producen en zonas transfronterizas, cuya desigualdad es un factor clave para comprender estos intercambios.

En el caso de la península coreana, las relaciones fronterizas están fuertemente marcadas por la idea de seguridad nacional. Una de las muchas consecuencias que tuvo el conflicto civil de los cincuenta fue la imposibilidad de establecer una zona transfronteriza en la península. No obstante, y pese al riesgo que implica la movilidad, la migración de ciudadanos de Corea del Norte a Corea del Sur es una realidad. Bulard (2016), en su estudio sobre refugiados norcoreanos en Corea del Sur, da cuenta de la desigualdad existente entre ambos países. Corea del Sur, al tener una mejor posición económica y diplomática, recibe constantemente ciudadanos de Corea del Norte, quienes buscan asilo en el país vecino. La vida que mantienen los inmigrantes norcoreanos, después de los tres meses de reclutamiento en Hanawon, está marcada por trabajos estandarizados para los inmigrantes. Los entrevistados de Bulard (2016) señalan: "Nosotros, los norcoreanos, solo tenemos los empleos que los locales rechazan" (p. 35). Las impresiones muestran una decepción en las expectativas, sin embargo, la movilidad existe pese a las dificultades, ya que la migración sigue siendo una mejor opción. Cabe mencionar, que gran parte del comercio exterior de la república está destinado a la región asiática, dando cuenta de la importancia de una cooperación regional en la economía nacional.

Hasta ahora, se ha trabajado el término frontera apuntando a casos regionales y sus situaciones locales, sin embargo, la relación entre América Latina y Corea del Sur no es un caso de frontera regional, sino que está caracterizada por su rasgo transpacífico y/o intercontinental. Ya desde los inicios de la globalización, la idea de la caída de las líneas fronterizas ha ido cobrando posición en el proceso de internacionalización del consumo. Muchas fuentes académicas hablan de la importancia de las redes sociales y de los medios masivos de comunicación en la exportación de la cultura surcoreana a América Latina. Madrid-Morales y Lovric (2015) comentan que, en el caso de la industria musical, las reproducciones de canciones del K-pop traducidas al español suman millones de visitas en la plataforma Youtube. Min, Jin y Dal (2018) comentan que, en el caso de Chile, los fans del K-pop tuvieron su primer acercamiento mediante Youtube o por recomendación de amigos, siendo las redes sociales un factor clave de ese intercambio. En el caso general de Latinoamérica, ocurre algo diferente, ya que el acercamiento hacia la música tiende a ocurrir después de un primer encuentro con la industria televisiva $(2018$, p. 4). La cultura popular surcoreana, atravesando fronteras físicas, idiomáticas e idiosincráticas, se posiciona de manera sobresaliente en el mercado internacional y en los medios masivos de comunicación. MadridMorales y Lorvic (2015) problematizan también la idea de "satisfacción de 


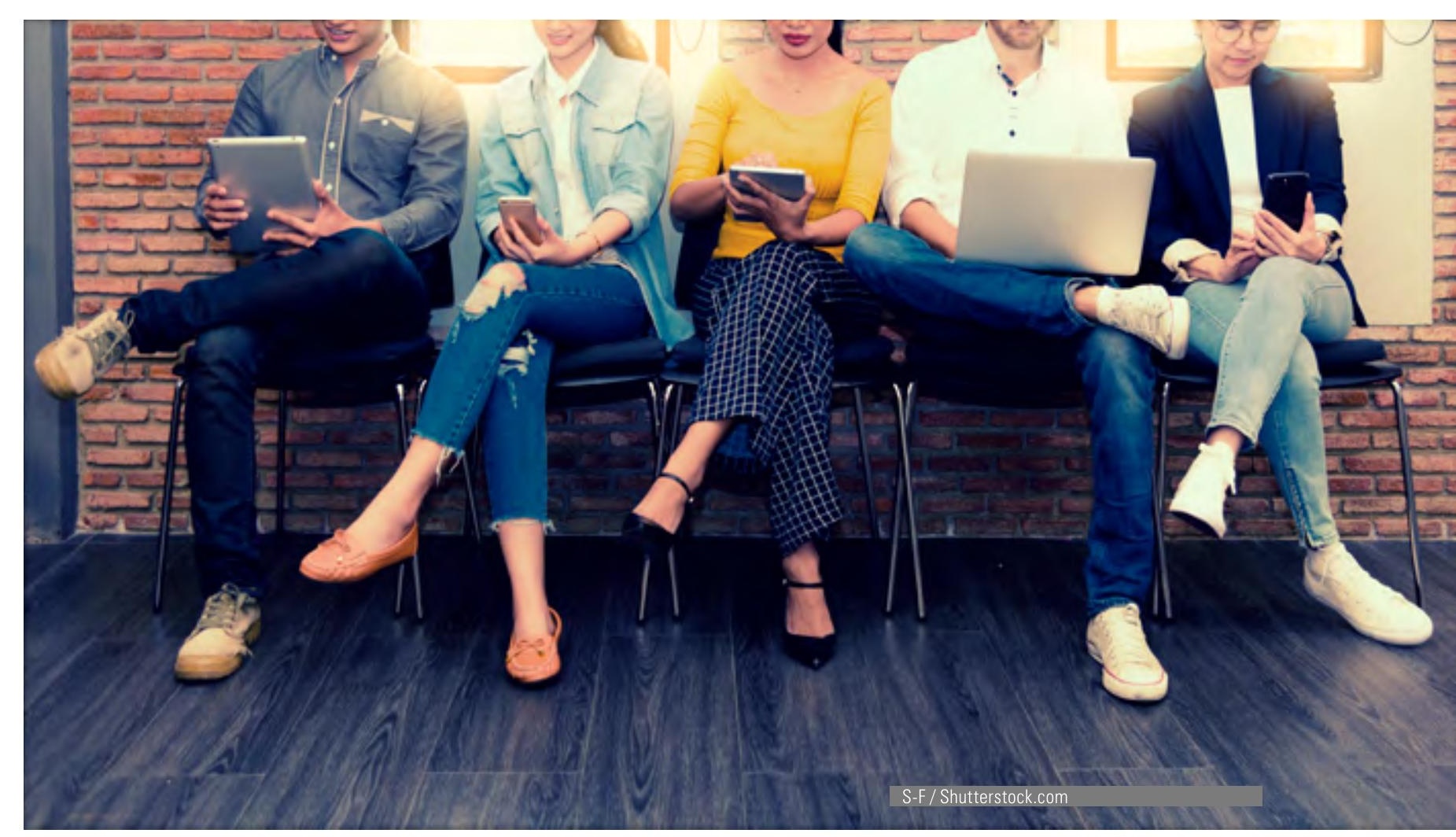

necesidades" propuestas por Ang (1985); y Blumler y Kazt (1974), comentan que las personas, al ser parte activa de los medios, integran una dinámica de actuación-recompensa. Bajo esta lógica, tanto la participación en línea como la adquisición de mercancía, además de contribuir en el intercambio desigual, brindan a los usuarios una posición social dentro de la esfera pública, es decir, les permite la proyección de una imagen social.

\subsection{Multiculturalismo}

Los Estudios Culturales son una entrada a la comprensión de los procesos contemporáneos de movilidad e intercambio. Jameson (1993) se refiere a ellos como una cuestión de "doble ciudadanía", un esfuerzo por combinar y/o coordinar varias identidades al mismo tiempo (Jameson, 2017, p. 78). La condición híbrida a la que está ligada el sujeto moderno es, en gran parte, consecuencia del juego competitivo de las economías mundo, en el que inevitablemente hay perdedores y ganadores (Jessop, 2004). Bajo esta premisa, el Korean Wave debe ser entendido como el resultado de la victoria que tuvo la República de Corea.

La coexistencia dinámica de las culturas, a través de los medios de comunicación, deja al descubierto la presencia masiva del capitalismo como organizador de una nueva realidad. La presencia de la cultura popular coreana en América Latina responde a la emergencia de este multiculturalismo sintomático en la economía. Žižek (1997) se refiere a una "emergencia de lo opuesto", en la medida en que el lado receptor es entendido como el perdedor en la competencia por el dominio del mercado. Vale decir, parte del triunfo del Korean Wave en Latinoamérica es producto de la derrota de las economías locales en la lucha internacional. Algunos ejemplos de esto son la imposibilidad de solventar economías desarrolladas, los índices de 
desigualdad social —en los que Latinoamérica aparece como una de las regiones más desiguales del mundo (Gilbert, 1998)—, incluso el rechazo a la cultura local por parte de los propios ciudadanos: "Another respondent praises Korean society by saying that it is 'superior to ours'" (Madrid-Morales y Lorvic, 2015, p. 35). La idea del éxito de una cultura, así como el trabajo en una imagen internacional sólida, son factores que impulsaron el éxito del Hallyu. Grüner (1998) adjudica valores ideológicos y culturales a las modas, comenta que no son solo la prueba del fetichismo de la mercancía - como diría Marx - en la globalización, sino que también representan las ideas centrales del capitalismo, además de las características propias de los Estados potencia en la economía mundo.

Žižek (1997), en su estudio sobre el multiculturalismo, se detiene a reflexionar sobre el ciberespacio y su impacto social. El autor relaciona la economía del mercado capitalista tardío a la experiencia individual de los consumidores en la Red, esto ocurre en la medida en que hay una repercusión directa sobre el sujeto social. Es decir, existe una mediación en la plataforma digital dada por las redes de poder que gobiernan la globalización. El capitalismo libre de fricción que expuso Bill Gates (1995) intenta acoplarse al éxito de esta nueva realidad, por eso se puede inducir que el anonimato, y la facultad creadora que ofrece el ciberespacio, rompen con la marginalidad de las clases sociales del mundo material. No obstante, un mundo sin esa fricción del capital quedaría anexado a las realidades fantasmagóricas que intentan proclamar los idealistas. Žižek hace énfasis en que el ciberespacio pretende liberar a los usuarios de las rivalidades que marcan el intercambio social, los posiciona en un escenario abstracto "en el cual se borra la particularidad de la posición social” (2017, p. 142).

En el caso del Hallyu, la idea de cercanía a los artistas, así como a la 'imagen moda' que se ofrece mediante el ciberespacio, está condicionada a una adquisición material y a la exposición de la misma. En los países latinoamericanos, los consumidores de esta cultura participan activamente en discusiones y grupos relacionados a la moda coreana. En el estudio de Min, et. al (2018), se presentan casos de fans, que para poder ser más activos en las diferentes plataformas web, comienzan a estudiar el idioma coreano: "All the participants were enthusiastic about Korean pop culture, and some had been involved in off-line fan activities and had to learn the language" (2018, p. 4). En el caso de Chile, los grupos de fans de K-pop popularizaron un recinto municipal por ser alberge de cientos de fanáticos que acuden a practicar coreografías y producir material para las redes sociales (2018, p. 2). En este último ejemplo, se desplazada la inmaterialidad, pues la reunión social in situ no solo proclama territorio, sino que también es punto de creación material para el consumo en la Red.

Žižek (1997) sostiene que el Estado-Nación es la forma social predominante del "universal concreto", es decir, el Estado es el que dirige las identidades de los individuos dentro de la sociedad (Žižek, 2017, p. 155). Y en esa organización de la población está supuesta la existencia de una "lealtad fundamental" —en términos hegelianos_ - por parte de los ciudadanos; lealtad determinada por la cultura que defiende la nación. Hegel afirma que la única manera que el individuo tiene para apartarse de su comunidad es cambiando esa lealtad fundamental, "reconocer la sustancia de su ser en otra comunidad, secundaria, que es a un tiempo universal y 'artificial', no 'espontánea' sino 'mediada', sostenida por la actividad de sujetos libres independientes" (Hegel, s.i. en Žižek, 2017, p. 154). En relación a lo expuesto por Žižek, es posible establecer una correspondencia con el Korean Wave en Latinoamérica, planteándose como base el cambio de la "lealtad fundamental" por parte de los seguidores de la cultura surcoreana. Resulta complejo aceptar que los fans arriesgan su imagen pública en pos de defender una nueva lealtad hacia una segunda cultura, pa- 
sando por alto ordenamientos estandarizados como los que plantea el modelo de cortesía de Brown y Levinson (1987). "A majority feels that the rest of society looks down upon them, that they are judged for emulating Korean styles and criticized by their environment" (Madrid-Morales y Lovric, 2015, p. 36). El multiculturalismo, al ofrecer una gama de diversas culturas, aceptadas y respetadas, plantea el dilema de la identidad nacional en un orden neofundamentalista, así como también el de distancia entre las culturas en el imperio del capital.

\section{4. Dinamismo en la esfera pública: construcción de la imagen (face)}

Hanna Arendt (2003) explica que la frontera entre la esfera pública y privada está situada en un escenario ambiguo, y que su división se ha convertido en algo insignificante. La participación del individuo en los "asuntos del mundo", todavía dependiente del componente material, ha construido una realidad situada en la esfera pública, la cual, durante un tiempo, fue considerada némesis de la esfera privada. Es decir, se planteaba una dicotomía entre el "yo real" y la "apariencia", haciendo de esta última la imagen que el individuo presenta en la sociedad.

Brown y Levinson (1987), en su estudio sobre la teoría de la cortesía, mencionan el concepto de face (imagen) como una construcción social que los hablantes deben proteger. Los autores, basados en los estudios de Leech (1983), indican que la valoración coste/beneficio que realiza el sujeto es lo que determina el grado de cortesía que utilizará.

Bajo este contexto, los estudios de Leech (1983) proponen dos tipos de cortesía: por un lado, la cortesía positiva, que pretende maximizar la cortesía de las ilocuciones corteses; y la cortesía negativa, que consiste en minimizar la descortesía de las ilocuciones descorteses (1983, p. 84). Escandell (2003) agrega que el lenguaje, al ser una construcción de interacción, no puede ser desentendido del componente social. Por lo tanto, la cortesía debe entenderse también como regulador del comportamiento social. El hablante, dependiendo del contexto, utiliza competencias sociopragmáticas para proteger su imagen en la situación comunicativa, ya que esta acción se considera necesaria para garantizar un equilibrio en las comunidades.

Kim y Nam (1998) proponen que, por un lado, en Asia el concepto de face está fuertemente vinculado a su organización social colectivista. Vale decir, el esfuerzo de encajar en el grupo asignado, y reprimir las individualidades, es lo que protege la imagen pública del hablante. Por otro lado, los autores mencionan que en occidente el método individualista es el que prima a la hora de la acción comunicativa. En este caso, los participantes trabajan en potenciar su imagen, es decir, evidenciar aquello que los hace diferente. América Latina, como heredera del modelo individualista — por un tiempo aspirante a un sistema colectivo-, desarrolla un ambiente en el que las personas buscan grupos donde encajar, pero al mismo tiempo, sufren la necesidad de resaltar en esos colectivos. Es entonces cuando el tema de la identidad cobra valor en diferentes dimensiones sociales; por una parte en una escala individual, y por otra, en una escala nacional.

Gruner (1998) sostiene que los neofundamentalismos son el fenómeno más enigmático de la posmodernidad globalizada, estos representan la búsqueda de nuevas identidades, aunque a su vez, parecieran ser la búsqueda de una identidad previa que se perdió (Gruner, 2017, p. 43). El rol de la apariencia, en relación a la identidad, pareciera cobrar un valor mimético —en términos platónicos- La verdad, como principio moralizante, se cuestiona desde la teoría multiculturalista, dando pie a un mundo lleno de representaciones. Gruner expresa la idea de que "el mundo sangriento y desgarrado en el que vivimos es pura ficción, un mero simulacro" (2017, p. 38), la dualidad 


\section{Reflexiones en torno al imperio de la apariencia y el orden multicultural}

Existe una polarización entre máscara e identidad en el imaginario colectivo. La idea de atribuir la connotación de falso a la máscara, y condenarla a un mundo ilusorio, es la carga que también se pone sobre la palabra "apariencia". Gianni Vattimo, ya en 1992, proclamaba el ocaso del mundo en el que el "ser" y el "parecer" se entendían como antónimos. La sustancialidad de la metafísica tradicional ya no existe más en el juego de las apariencias, que es ahora el nuevo mundo (1992, p. 22). Muchas de las críticas que se hacen en la esfera pública, respecto a la apariencia como máscara, están asociadas a la idea de usurpación y falsedad. No es de extrañar, entonces, que a los seguidores del Korean Wave se les critique y menosprecie en las culturas locales. La idea de defender la "identidad nacional" todavía juega un rol importante, sin embargo, y como adelanta Jossep (2004), los Estados Nacionales están en crisis desde hace años, y muchos de ellos ya se han descompuesto (2004, p. 36). El orden hegemónico que propone la identidad nacionalista ha sido quebrantado y fuertemente cuestionado en el mundo moderno.

Corea del Sur, desde la segunda mitad del siglo XX viene trabajando en el fortalecimiento de su economía y en el papel que desea en el mundo, optando por un capitalismo dirigido en el esquema socioeconómico interno. El reforzamiento que puso el Estado en la exportación de cultura, debe ser entendido como una estrategia clave para el éxito del Korean Wave en el mundo. Lee (2011) pone énfasis en que la cultura es el elemento más importante para la creación de una marca de imagen nacional (2011, p. 89); además, se debe añadir el factor económico, pues Corea del Sur se ha posicionado, de manera paulatina, en escalas superiores. Creusa Muñoz (2016), en concordancia con lo anterior, comenta que Corea del Sur, en su esfuerzo por defender su papel de potencia en el mundo, lucha mediante dos tipos de armas: la económica y la cultural. El triunfo de esta estrategia en América Latina da cuenta de la fuerza con la que el país asiático supo defender su puesto. Y hoy en día, la relación intercontinental que se genera entre ambas partes es la evidencia de dicho proceso.

En América Latina, iniciado el proceso de desnacionalización de los Estados, surge con fuerza la idea de independencia del individuo social. Es decir, bajo la suposición del quiebre del símbolo estatal, los ciudadanos se convencen de su autonomía en la esfera pública. Oliver Costilla (1998) reflexiona en torno a la fragilidad de la hegemonía burguesa latinoamericana y en cómo su subordinación a fuerzas externas repercute inevitablemente en los derechos históricos y políticos de las poblaciones (1998, p. 56). El autor menciona que, como resultado de esta crisis hegemónica, aparece un "protoestado transnacional" que tomaría el lugar del Estado local. En este último punto, resulta evidente que Oliver Costilla se refiere a la incidencia norteamericana en Latinoamérica, no obstante, el análisis es aplicable a la influencia cultural de Corea del Sur en esta parte del continente; ya que las bases propuestas por el autor apuntan, principalmente, a un poder local "mediador" frente al dominio de organismos financieros transnacionales. El poder que ejerce la cultura en este aspecto da cuenta de una debilidad en la hegemonía burguesa latinoamericana, la que — según el autor- fue la clase dominante del modelo capitalista en la región. El mismo Oliver Costilla reconoce que el dinamismo de las culturas no respeta un patrón específico, sino que muy al contrario, fluyen y se desplazan por rutas "singulares, inimaginables e influyen en la propia reproducción social" (1998, p. 44). Dicho dinamismo es el que ocurre con el Korean Wave en la región latinoamericana, desde su llegada con la exportación de dramas televisivos, hasta la creciente popularidad de la música, gastronomía, idioma y moda. La débil idea del prestigio nacional, además de 
Ios años de adoctrinamiento al modelo neoliberal tras el fracaso socialista, han permitido un terreno perfecto para la recepción de nuevas culturas. Y ciertamente, la gran valoración que el éxito económico de Corea del Sur proporciona a su cultura, ha influenciado en la percepción de su imagen.

\section{1. Discusión de la dicotomía apariencia - identidad en la modernidad}

El concepto identidad siempre ha generado discusión, pues su complejidad responde a un sistema multiforme que reafirma su poder mediante constantes mutaciones que no pierden fuerza a través del tiempo. Los estudiosos, durante siglos, han intentado desglosarlo y establecer una taxonomía que pueda guiar la idea de identidad a una realidad más concreta. Situado en esta discusión, el filósofo ruso Mijaíl Bajtín (1895-1975), a lo largo de toda su obra, estableció una relación dependiente entre el 'yo' y el 'otro', influenciando así los estudios antropológicos y sociológicos sobre el concepto de identidad (Bubnova, 2000). Para Bajtín (1997), el espacio entre el yo y el otro es el lugar en donde se "distribuyen y disponen todos los momentos concretos del ser" (1997, p. 79). Es entonces que se piensa la identidad como parte de una construcción social. La apropiación de ese espacio comunicativo supone interacción, y en ese acto de reconocimiento surge el 'ser' como una entidad realizable y verosímil. Alejos García (2006) agrega que es crucial pensar en el otro como un factor inamovible de la realización del individuo, "el otro precede al yo, lo alimenta e instruye, lo acompaña toda la vida" (2006, p. 48). La alteridad —entendida desde la teoría bajtiana—, no solo valida la identidad del individuo, sino que además aprueba y defiende la imagen pública — facede un país (en este caso, Corea del Sur). La relación de dependencia articula un despertar de conciencia entorno a la función del 'testigo' en la comunicación. En este sentido, la apropiación de espacios cibernéticos se convierte en una parte clave en la construcción moderna de identidad, pues el testigo cumple una función sustancial para el principio realista de verosimilitud, así como también para la construcción de la apariencia en la esfera pública.

El ciberespacio, como ya se ha dicho, es un lugar importante para el consumo de cultura, así como también para la expresión de la misma. Es innegable que el dominio de las redes sociales ha escalado a niveles impresionantes. Ejemplo de esto es el impacto de YouTube para la industria

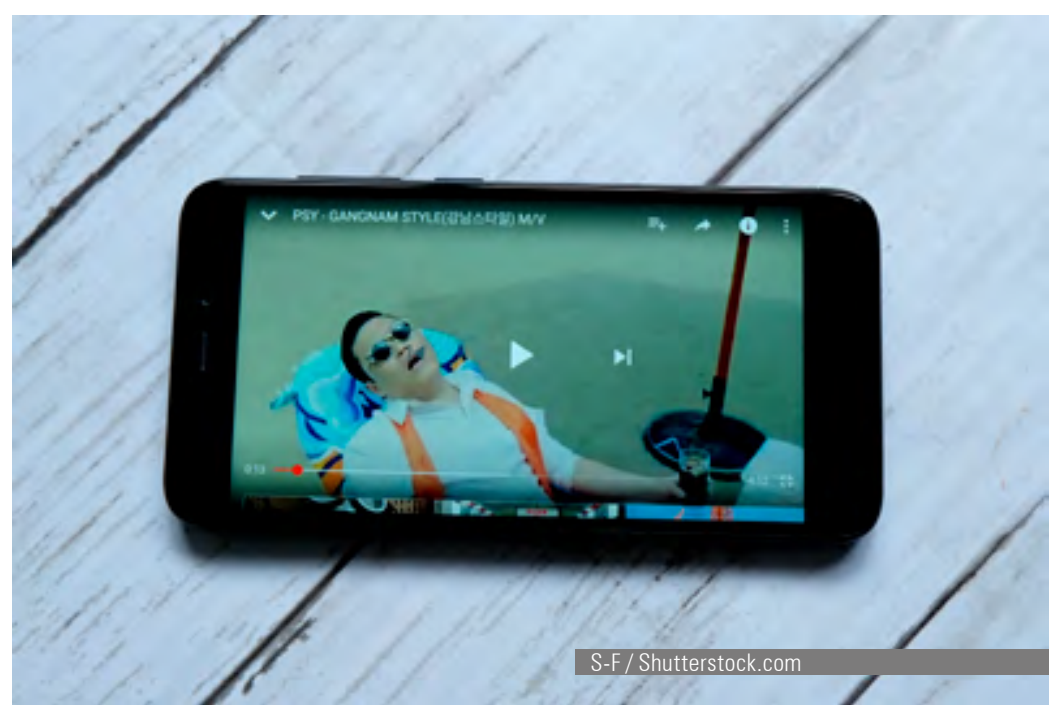


musical. "According to research conducted by FTI Consulting, there are approximately 259 million Facebook users and 405 million monthly unique YouTube users in Latin America" (Punt, 2018 en Min et. al, 2018). No es casualidad que las plataforma web asuman un rol fundamental al momento de mencionar el respaldo de América Latina hacia los ídolos del Korean Wave. Además del consumo de música y cultura, como ya se ha mencionado, está presente también la creación de contenido nuevo. El espacio virtual, al brindar herramientas de construcción social, propicia un escenario de integración de los participantes; dando como resultado proyectos de apoyo a los artistas y material de difusión de la cultura coreana (reproducciones de videos, traducción de letras, reacciones e impresiones, etc.). La invisibilidad y la exposición coexisten en un medio que era antes identificado por el anonimato. Como consecuencia, la apariencia se presenta nuevamente como un orden imperante, pese a las críticas que los fundamentalistas exponen respecto a la máscara y su vínculo con la sociedad virtual.

El vacío universal del cual habla Žižek (1997) es lo que para Nietzsche representaba el mundo como fábula, es decir, la disposición completa del sujeto a moldearse y modificarse, a crear una apariencia y consentir que la superficialidad es un valor real y no carente de significación. Ya que, al no haber ningún ser verdadero, en palabras de Vattimo, esta creación novedosa del individuo no puede ser degradada a la mentira o a la ilusión. La verdad de esa imagen es la que los fans aprecian en sus artistas, el esfuerzo por preservar la apariencia y el efecto de identificación mediante la cercanía que brinda el ciberespacio. Arendt (2003) ya adelantaba el desplazo de lo doméstico hacia el campo del "interés colectivo", pues se ha dejado de pensar en la palabra 'privación' cuando se hace referencia a lo privado; la autora atribuye gran parte del mérito al individualismo moderno, no obstante, y como ya se ha mencionado antes, pareciera que el Korean Wave no se agota solo en el individualismo. La exclusividad de lo privado, entonces, existiría solo en el imaginario romántico moderno, que plantea al individuo como una esencia, y a su imagen pública como una apariencia ficticia y engañosa. Oscar Wilde admitió que el misterio del mundo está en lo visible y no en lo invisible; y es ese espacio en blanco, que se desconoce por naturaleza humana, lo que mantiene el equilibrio entre las esferas pública y privada. El Hallyu permite que el sujeto social adopte la cultura con mayor prestigio, que la incorpore y defienda, que pueda jugar con el efecto enceguecedor de la belleza y explore en una realidad distante que con el tiempo volverá cercana.

Gianni Vattimo (1992), en su estudio sobre la filosofía de Nietzsche y Heidegger, interpela el pensamiento fundamentalista que protege la idea de oposición entre esencia y apariencia. El autor, convencido de la premisa "el mundo verdadero es fábula, no existe ya ningún ser verdadero" (p. 11), nombra a la apariencia como nuevo eje estructurador de la esfera pública y privada. Al ser desplazado el sujeto real, la confección estética de la apariencia deja de ser sinónimo de falsedad. El individuo es libre de explorar y crear, de jugar con la plasticidad y frivolidad del mundo. Entonces, la adopción de una estética identificada como 'extranjera', como lo es el Korean Wave en América Latina, no puede ser valorada como mímesis platónica, sino como identidad pura. La fragilidad de la imagen nacional en los países latinos, no logra competir con la imagen que Corea del Sur ha realizado en su interacción con la alteridad (en este caso, Latinoamérica). Willoughby (2006) menciona que en las bandas surcoreanas se hace un trabajo exhaustivo en la imagen de los integrantes. El académico indica que la imagen es considerada como uno de los factores más importantes para los artistas; incluso más que la creatividad, la habilidad musical o el talento. La importancia de la imagen es más que una fijación estética general; esta debe ser entendida también como el punto de conexión entre el artista y el seguidor. Los fans, al sentir identificación con 
su ídolo, o al reconocer en él un rasgo similar al propio, refuerzan la idea de identidad con esa segunda cultura.

\section{Conclusiones}

En el presente estudio se han abarcado temas tales como la globalización, el rol de los Estados en la mundialización del capital, las fronteras, el multiculturalismo, el concepto imagen (face) y la dinámica que se ejerce en la esfera pública del mundo contemporáneo en relación a la dicotomía imagen/apariencia. El Korean Wave, como foco de la discusión, se ha entendido como el resultado del éxito económico de Corea del Sur, así como también de la estrategia que dicho país ha realizado desde la segunda mitad del siglo XX para expandir su poder mediante dos herramientas: la transición desde el capitalismo dirigido hacia la liberalización económica en la época de regímenes militares y democratización; y el uso de soft power en la escala internacional tras adquirir estabilidad en la economía mundo. Ahora bien, en cuanto a las respuestas de América Latina frente a esta situación, las reflexiones abarcan desde la imposibilidad de competencia por parte de las economías latinas, debido a una inestabilidad económica en la región, hasta las secuelas existentes en los Estados modernos producto del proceso de desnacionalización.

La cultura popular coreana, dotada del prestigio que su economía tiene a nivel global, se ha instalado en América Latina con una imagen pulcra, invitando a los ciudadanos de la región a incorporarse en un nuevo orden social. La apariencia, como componente crucial de las relaciones sociales, es influenciada por el reconocimiento de una cultura dominante; dejando en evidencia un cambio en orden jerárquico de las culturas; es decir, el podio utilizado históricamente por el mundo europeo y anglosajón ha cambiado en los últimos años. La creciente desaprobación al gobierno de Estados Unidos, y el bajo perfil de las naciones Europeas, han favorecido al crecimiento del Korean Wave en la región Latinoamericana. El trabajo que la potencia asiática ha puesto en el acercamiento de su cultura, además del cuestionamiento de la distancia —en términos de John Fiske_-, ha permitido la exploración del

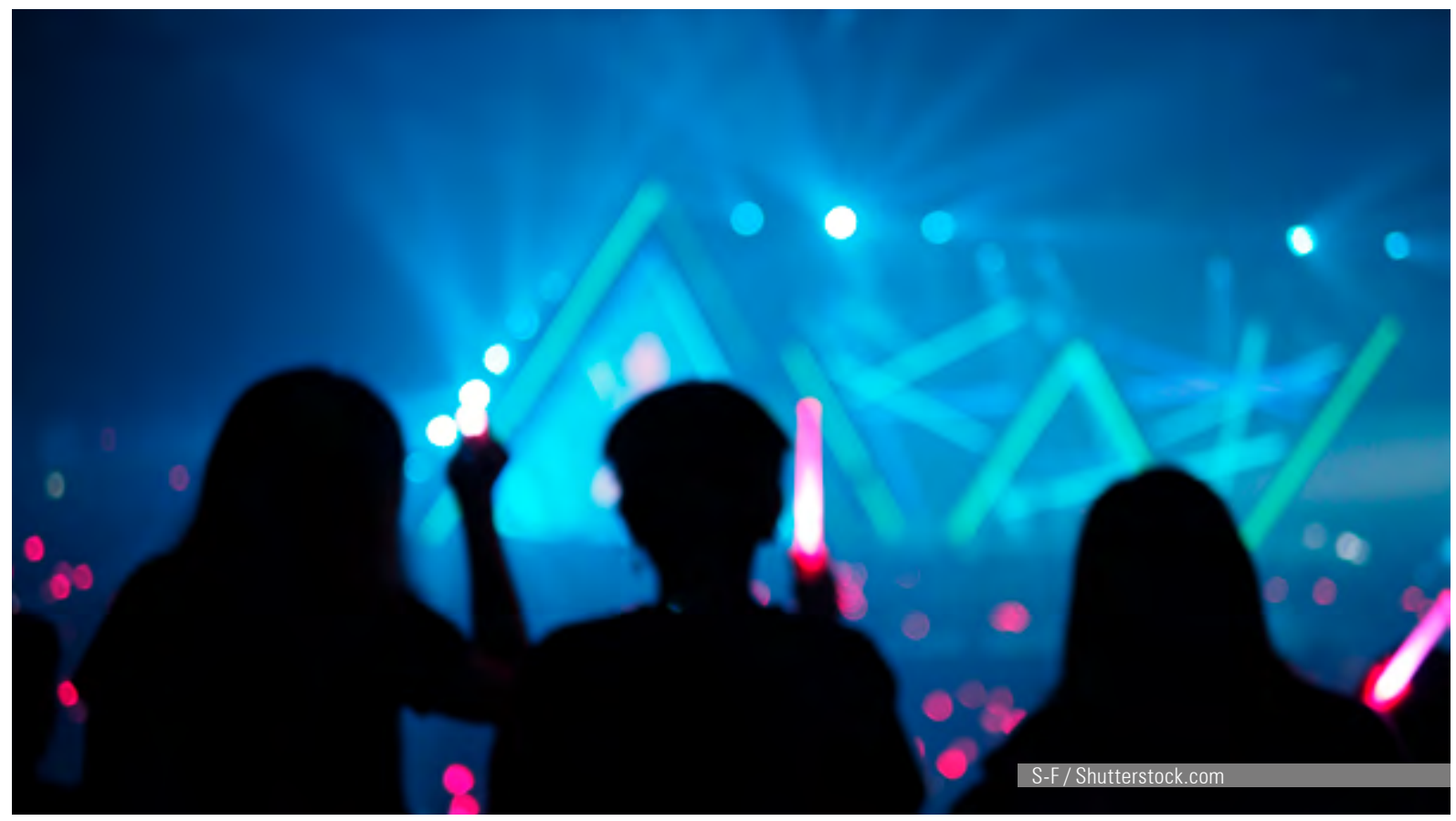


individuo en nuevas formas de identidad, e incluso en el cambio de la lealtad fundamental de su cultura. Como consecuencia, surgen también críticas en torno a la idea de apropiación cultural en la expansión de la marca del Hallyu.

En el punto 2.2 se mencionó que el intercambio desigual es un factor importante para la solvencia del Hallyu en América Latina. Ya que sin esa característica específica, el dinamismo entre ambas culturas sería de una forma muy diferente. En un escenario equilibrado de las economías, la cultura local trataría de impedir el abandono de la lealtad de sus ciudadanos. En este sentido, es importante recordar que la industria cultural trabaja con la idea - en cierto modo - ilusoria de identidad local, planteando un orden hegemónico y jerarquizado que valide el prestigio de los líderes. No es de extrañar que a los seguidores del Korean Wave les cueste identificar superficialmente las características particulares de esa moda. Madrid-Morales y Lorvic (2015) mencionan que la industria del K-pop y el K-drama se pueden catalogar como un excelente resultado de "hibridación", ya que lograron crear un producto exitoso que contiene elementos de diferentes culturas. Ahora bien, ¿es posible desacreditar la patente del Korean Wave abogando por una crítica de apropiación cultural? ¿Cuál es el rol que cumplen los artistas internacionales (no coreanos) en la imagen del Hallyu bajo el lente de la aculturación?

Como última reflexión, mencionar que este mundo, dominado por la globalización del mercado, ha desarrollado una forma de autoprotegerse. La creciente idea de revolución cultural en el espacio público, como forma de intimidación a la hegemonía global, es un método estratégico del modelo Capitalista, y no una forma de amenaza hacia él. ¿Es posible salir de ese esquema y buscar una alternativa? La autoregulación del modelo imperante es un tema sugerente para una futura investigación, así como también las interrogantes respecto a la apropiación cultural en el Korean Wave. Lo cierto es que los estudios culturales albergan una belleza que muchas veces solo puede ser contemplada desde la ceguera innata del ser humano. Reconocer un orden natural consiste también en aceptar la imposibilidad de alcanzar una verdad absoluta. 


\section{Referencias}

- $\quad$ Alejos García, J. (2006). Identidad y alteridad en Bajtín. Acta Poética, 27 (1), pp. 45-61.

- $\quad$ Ang, I. (1985). Watching Dallas: Soap Opera and the Melodramatic Imagination. London: Methuen.

- Arendt, H. (2003). La condición Humana. Ramón Gil (trad). Buenos Aries: Editorial Paidós. (Obra originalmente publicada en 1958).

- Bajtín, M. (1997). Hacia una filosofía del acto ético. De los borradores y otros escritos. Barcelona: Anthropos Editorial, 1997.

- Blumler, J. G. y Katz, E. (edts). (1974). The Uses of Mass Communication: Current Perspectives on Gratifications Research. Beverly Hills: Sage.

- Brown, P. y Levinson, S. (1987). Politeness. Some Universals in Language Use. Cambridge: Cambridge University Press.

- Bulard, M. (2016). Reeducación capitalista en Corea del Sur. En Las dos Coreas. (pp. 31-38). Santiago: Editorial Aún Creemos en los Sueños. (Artículo recopilado de Le Monde Diplomatique, 2013).

- Bubnova, T. (2000). Prólogo en Yo también soy. Fragmentos sobre el otro. México: Editorial Taurus. Pp. 11-26.

- Dilla, H. y Breton. I. (2018). Las regiones transfronterizas en América Latina. Revista Latinoamericana. (51), pp. 15-37.

- Escandell, V. (2003). El estudio de la cortesía. Introducción a la pragmática. Barcelona: Editorial Ariel. 135-154.

- $\quad$ Gates, B., Myhrvold, N., Rinearson, P., y Domonkos, D. (1995). The road ahead.

- $\quad$ Gilbert, A. (1998). The Latin American City. Latin America Bureau, London.

- Grüner, E. (2017). El retorno de la teoría crítica: una introducción alegórica a Jameson y Zizek. En Estudios Culturales: Reflexiones sobre el Multiculturalismo (pp. 3-56). Desligamiento Ediciones. (Trabajo original publicado en 1998).

- Jameson, F. (2017). Sobre los “Estudios Culturales”. En Estudios Culturales: Reflexiones sobre el multiculturalismo (pp. 57-126). Desligamiento Ediciones. (Trabajo original publicado en 1993).

- Jessop, B. (2004). La economía política de la escala y la construcción de las regiones transfronterizas. Revista eure. 24(89), pp. 25-41.

- Kim, J.Y. y Nam, S.H. (1998). The Concept and Dynamics of Face: Implications for Organizational Behavior in Asia. Organization Science, 9(4), pp. 522-534.

- Kim, W. (2015). La educación como herramienta de la política industrial: el caso de Corea. En W. Min (Ed.), Estudios coreanos para los hispanohablantes: un acercamiento crítico, comparativo e interdisciplinario (pp. 205-220). Santiago, Chile: Ediciones UC.

- Lee, S. J. (2011). The Korean Wave: The Seoul of Asia. The Elon Journal of Undergraduate Research in Communications. 2(1), pp. 85-93.

- Leech, G. N. (1983). Principles of Pragmatics. Londres: Longman.

- Madrid-Morales, D. y Lovric, B. (2015). Transatlantic Connection: K-pop and K-drama Fandom in Spain and Latin America. The Journal of Fandom Studies, 3(1), pp. 23-41.

- Min, W. y Jin, D. y Han, B. (2018). Transcultural Fandom of the Korean Wave in Latin America: Through the lens of cultural intimacy and affinity space. Media, Culture and Society. 1(16), pp. 1-16.

- Muñoz, C. (2016). Una nación fracturada. En Las dos Coreas (pp.15-18). Santiago: Editorial Aún Creemos en los Sueños. (Artículo recopilado de El Exportador Corea. Bs. Aires, 2015).

- Oliver-Costilla, L. (1998). El Estado latinoamericano ante la mundialización del capital. Estudios Latinoamericanos, 5(9), pp. 39-65.

- Stanley, E.A. (2009). Ending the Korean War: The Role of Domestic Coalition Shifts in Overcoming Obstacles to Peace. International Security, 34(1), pp. 42-82.

- Shim, D. (2008). The Growth of Korean Cultural Industries and the Korean Wave. En B. H. Chua and K. Iwabuchi (eds.), East Asian pop culture!: Analysing the Korean wave. Hong Kong: Hong Kong University Press, pp. 16-31.

- $\quad$ Pipitone, U. (2003). Ciudades, naciones, regiones: Los espacios institucionales de la modernidad. México: Fondo de Cultura Económica. (pp.322-363).

- $\quad$ Punt DJ (2018) Mobile-first: Milleannials in Latin America. In: Proceedings of the National Association of TelevisionProgram Executives (NATPE) Conference, Miami, FL, Latin American.

- Riffo, L. (2003). Gobalización, metropolización y mercados de trabajo. En I. Trujillo (Ed.), América Latina: Globalización, metropolización y desigualdades sociales. (pp. 15-44). Santiago, Chile: Editorial ARCIS.

- Vattimo, G. (1992). Más allá del sujeto. Nietzsche, Heidegger y la hermenéutica. Juan Gentile (Trad.). España: Editorial Paidós.

- Willoughby, H.A. (2006). Image Is Everything: Marketing of Femininity in South Korean Popular Music, in K. Howard (ed.). Korean pop music: riding the wave. Folkestone: Global Oriental, pp. 99-107.

- Žižek, S. (2017). Multiculturalismo o la lógica cultural del capitalismo multinacional. En Estudios Culturales: Reflexiones sobre el multiculturalismo (pp. 127-176). Desligamiento Ediciones. (Trabajo original publicado en 1997). 